\title{
Maxillary labial frenectomy by using conventional technique and laser - Report of two cases
}

\author{
Archana N.V ${ }^{1 *}$, Jose Paul ${ }^{2}$, Johnson Prakash D’Lima ${ }^{3}$, Senny Thomas parackal ${ }^{4}$, Deepak Thomas ${ }^{5}$, Akhil S $^{6}$ \\ ${ }^{1,6}$ Post Graduate student, ${ }^{2}$ Professor and Head, ${ }^{3,4}$ Professor, ${ }^{5}$ Reader, Dept. of Periodontics, Annoor Dental College and \\ Hospital, Muvattupuzha, Ernakulam, Kerala, India
}

Corresponding Author: Archana. N.V

Email: drarchana119@gmail.com

\begin{abstract}
Aberrant frenum causes a problem of midline diastema which leads to unaesthetic smile for the patient. Orthodontic correction of midline diastema helps to create aesthetic smile but frenum attachment may interfere it. The labial frenectomy is the procedure performed to remove aberrant frenum attachment. This article presents two case reports of maxillary labial frenectomy by using conventional technique and Laser.
\end{abstract}

Keywords: Labial Frenectomy, Midline diastema, Aesthetic smile, Laser frenectomy.

\section{Introduction}

One of the more exciting but often misunderstood anatomic structures in the oral cavity is the frenum. It is a mucous membrane fold that attaches the lip and the cheek to the alveolar mucosa, the gingiva, and the underlying periosteum.

A frenulum is a small frenum. ${ }^{1}$ In a normal oral cavity several frena are usually present, most specifically the maxillary labial frenum, the mandibular labial frenum, and the lingual frenum. The main function of the frenum is to provide stability of the upper and lower lip and the tongue.

Content of frenum is;

1. Variable amount of loose connective tissue with elastic and dense collagen fibers

2. Fat cells

3. Occasionally acini of mucous producing salivary glands

4. Muscle fibers

5. Mucogingival junction - closely associated.

Classification of frenum by Placek et al $1974^{2}$

1. Mucosal: when the frenal fibres are attached up to mucogingival junction

2. Gingival: when fibres are inserted within attached gingiva

3. Papillary: when fibres are extending into inter dental papilla

4. Papillary penetrating: when the frenal fibres cross the alveolar process and extend up to palatine papilla.

Frenum is protected by stratified layered epithelium containing vascular structures with thin peripheral nervous ramifications. Hypertrophic, fibrotic, ample, fan-shaped or bifid ending construction are described as an abnormal frenum whose development is not dependent upon its point of insertion. ${ }^{3}$

Frenectomy or frenotomy procedures can be used to treat the abnormal frenum. Surgical removal of the abnormal frenum is known as frenectomy that includes its attachment to the underlying bone, whereas frenotomy is the incision and the repositioning of the frenal attachment. Frenectomy can be done either by the routine scalpel technique, electro surgery or via lasers. The conventional technique involves using a scalpel to excised the frenum. In fact, it holds the routine risks of surgery such as bleeding and patient compliance. $^{4}$

The role of laser in dentistry has been well established in traditional oral disease management. ${ }^{5}$ It is a device that can attain maximum oral health in a minimally invasive manner so far that laser technology is evolving very rapidly. ${ }^{6}$ The leading laser demonstrated by Robert N. Hall in 1962 remained a diode laser that stands as a solid-state semiconductor laser that utilizes a combination of Gallium $(\mathrm{Ga})$, Arsenide (Ar) and additional components, such as Aluminum (Al) and Indium (In), to convert electrical energy into light energy. Usually it is operated in contact mode through a flexible fiber optic delivery system that emits in continuous-wave or gated-pulsed modes. $^{7}$

\section{Case Report 1}

A 17 year old male patient presented with the chief complaint of spacing between the upper anterior teeth and the patient was referred from the department of orthodontics for frenectomy. 
Patient's medical history was not significant.

Intraoral examination showed papilla penetrating type hypertrophic labial frenum and midline diastema between maxillary central incisors.

After taking patient's consent it was decided to do frenectomy with classical technique by Archer (1961) and Kruger (1964).

\section{Armamentarium}

Scalpel blade no.15, Gauze pieces, Tissue forceps, Silk sutures, Hemostat, Scissors

The area was anesthetized with a local infiltration by using 2\% lignocaine with 1:200000 adrenaline

Fig. 1: Maxillary labial frenum (Papilla penetrating type) with diastema between maxillary central incisors.

Step 1: The frenum was engaged with a haemostat which was inserted into the depth of the vestibule and the incision with the 15 no scalpel blade was placed on the upper and the lower surface of the haemostat until the haemostat was free. The triangular resected portion of the frenum with the haemostat was removed, which leaves a rhomboid area exposing the deeper connective tissue fibers. (Fig. 2)

Step 2: Dissection of muscle fibers was done on the bone. (Fig. 3)

Step 3: The edges of the diamond shaped wound were sutured by using 3-0 black silk with simple interrupted sutures. (Fig. 4)

Fig..5: Showed postoperative view after one week.

\section{Case Report 2}

A 14 year old female patient presents with chief complaint of mild spacing between the upper front teeth and the patient was referred from the department of orthodontics for frenectomy.

No significant medical history reported

The clinical examination revealed the presence of papilla penetrating type hypertrophic labial frenum and midline diastema between the central maxillary incisors (Fig. 6)

The treatment of choice was to perform laser frenectomy after complete evaluation and detailed history.

Informed consent was obtained from the patient to perform frenectomy after explaining the intra and postoperative aspects.

The frenectomy performed with a wavelength of $980 \mathrm{~nm}$ with photon plus diode laser manufactured by Zolar Tech technology. (Fig. 7)

The laser was operated at a power of 3.0 watt in continuous wave mode with $400 \mu \mathrm{m}$ optical fiber.
Local anesthesia was administered and laser fiber was applied vertically and laterally to the frenum, which initially disrupted the continuity of the mucosa. (Fig. 8)

This made it easy to access in performing a deeper cut of the frenum in a horizontal dimension. (Fig. 9)

Wave length Specific goggles were worn by the patient and all the staffs throughout the procedure. And high speed evacuation was used to reduce the slight charred odor and to remove the laser plume.

Hemostasis was achieved without suturing

According to the report of the patient there was no post-operative complications

New insertion of labial frenum was observed at the mucogingival junction after 2 months post operatively. (Fig. 10)

Also improved oral hygiene with easy access to the cervical region was claimed by the patient.

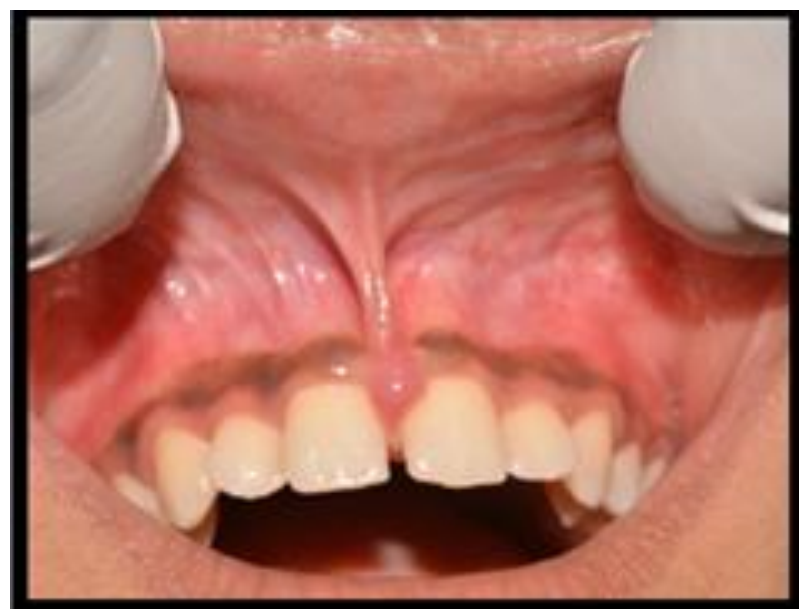

Fig. 1: Preoperative

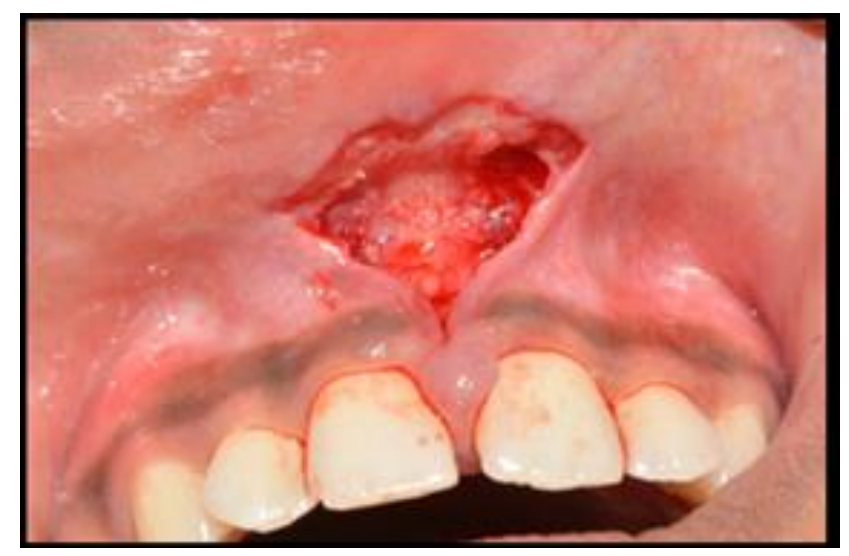

Fig. 2: Appearance after the initial incision and frenum removal 


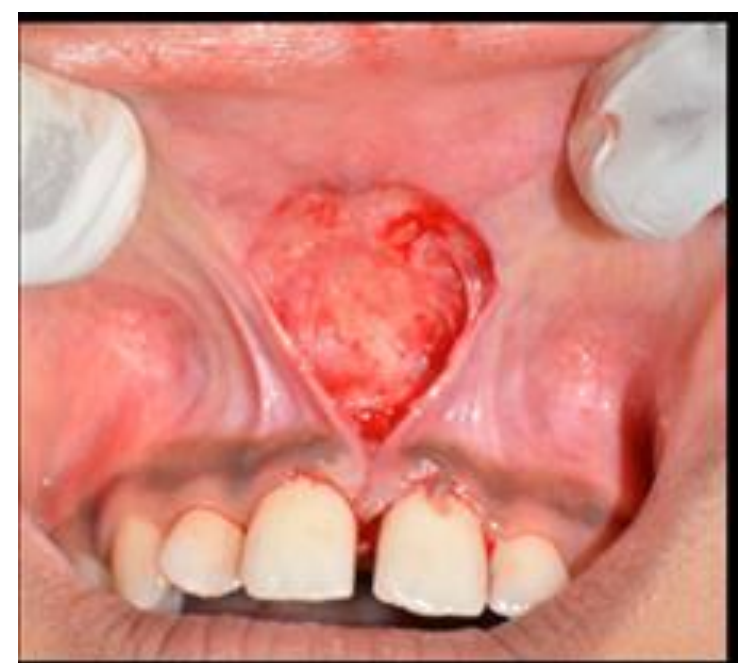

Fig. 3: Appearance after the dissection of muscle fibers

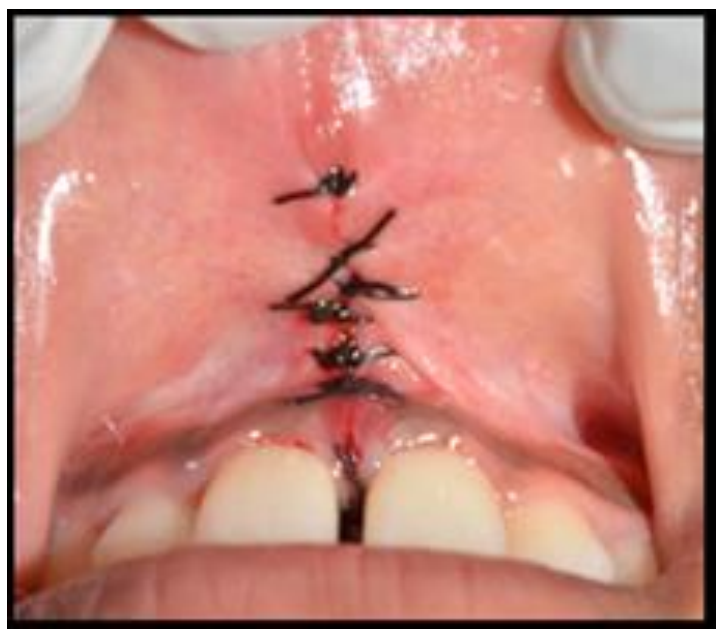

Fig. 4: Simple interrupted sutures given

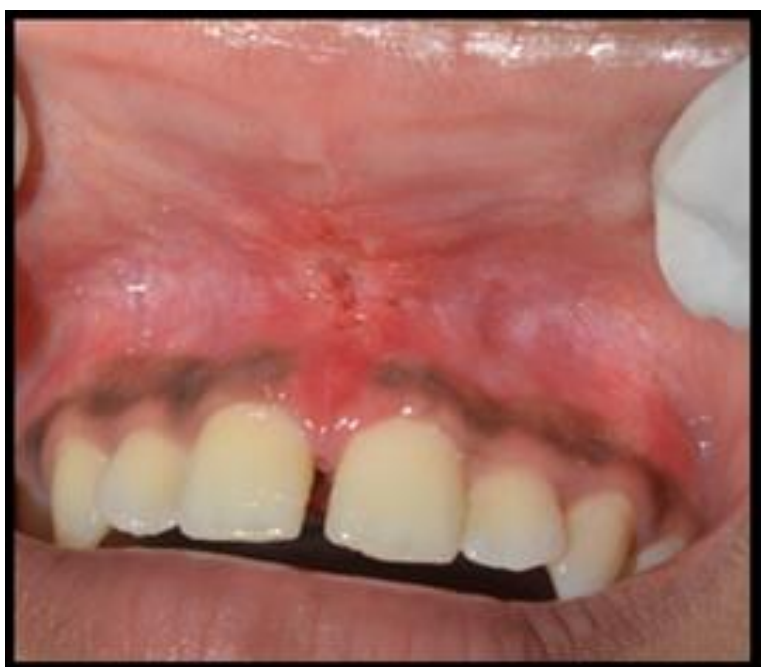

Fig. 5: one week post operative

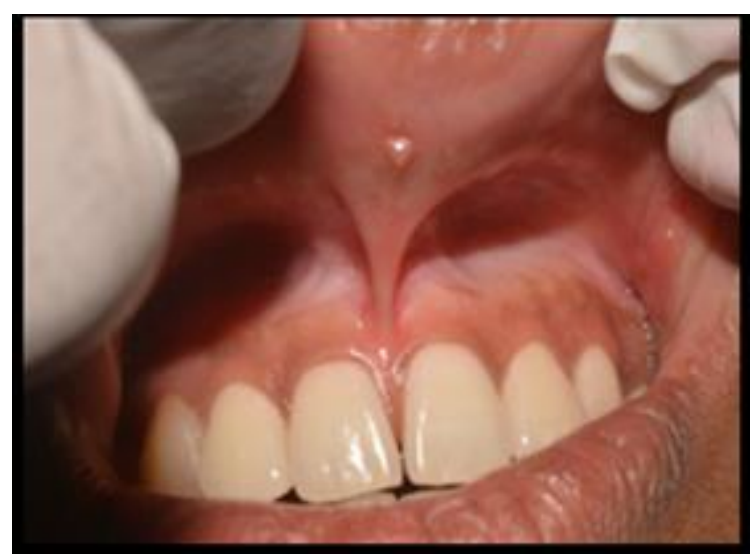

Fig. 6: Preoperative

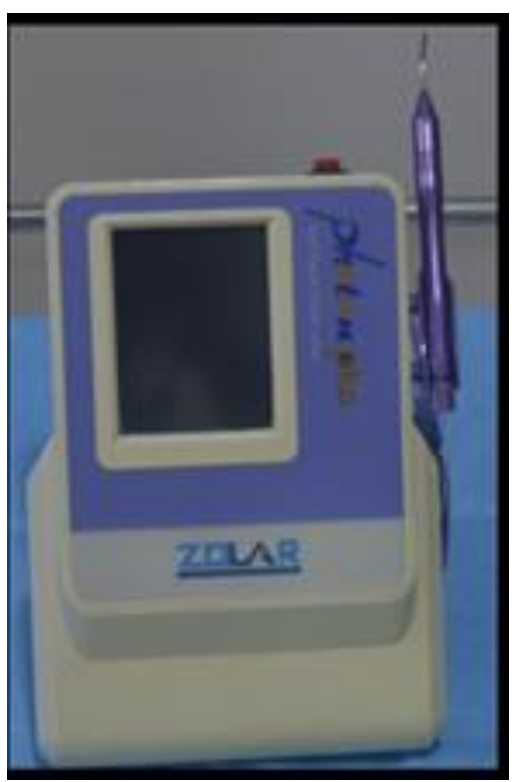

Fig. 7: Zolar Photon plus diode laser

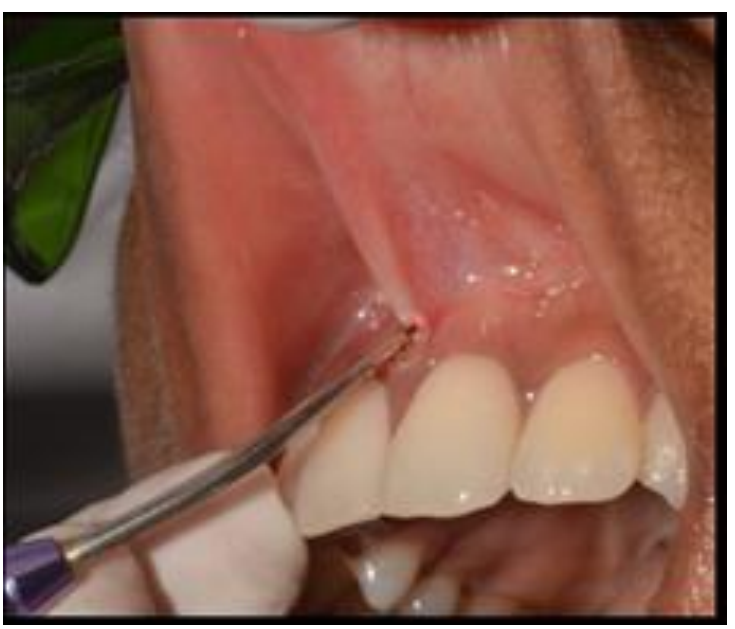

Fig. 8: Laser was applied 


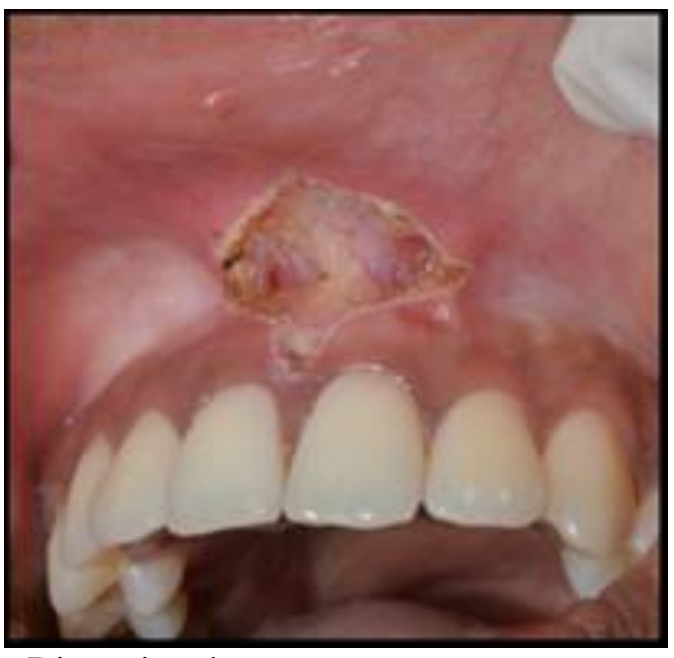

Fig. 9: Dissection done

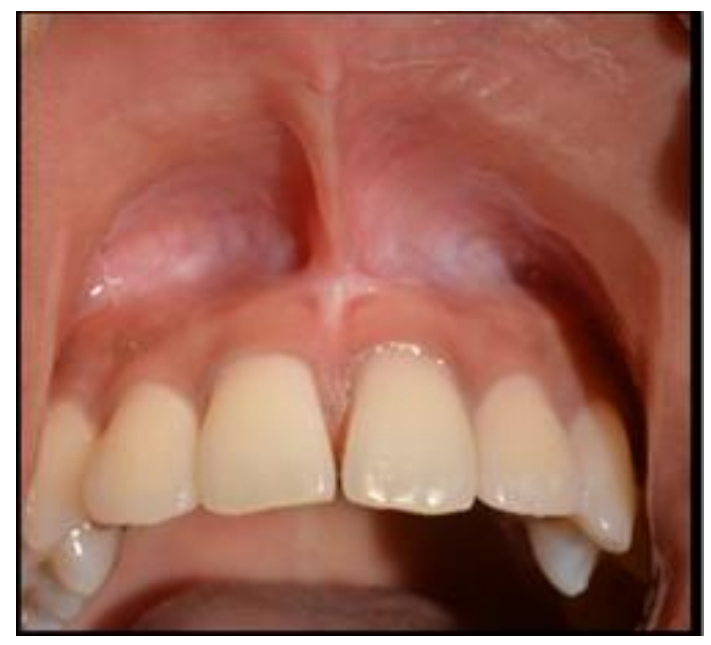

Fig. 10: Two months post operative

\section{Discussion}

There has been much controversy over the relationship between the maxillary midline diastema and the labial frenulum. Before 1940, Frenectomy was considered prior to orthodontic treatment. ${ }^{8}$ The primary cause of the diastema was regarded as Frenulum. Broadbent ${ }^{9}$ later showed that after teeth eruption the problem is self-correcting. In his examination of 6-year-olds, Taylor $^{10}$ found that $98 \%$ had a midline diastema. By age 12 , only 7 percent had a persistent diastema, thereby confirming Broadbent's findings.

Surgical correction is currently recommended after orthodontic treatment and eruption of permanent anterior maxillary teeth. It may be due to the difficulty of moving teeth through scar tissue and the problem's self-correcting nature. Instead of various modifications, a classical frenectomy is widely followed. The classical technique results in a longitudinal surgical incision and scarring, that can lead to various periodontal problems and an unesthetic appearance, thus mandating further modifications. ${ }^{\mathbf{1 1}}$

The laser is a viable alternative for conventional techniques for many intraoral soft tissue surgical interventions. The commercially available dental instruments have wavelengths varying from $488 \mathrm{~nm}$ to $10,600 \mathrm{~nm}$ and are completely non-ionizing radiations to avoid any mutations in cellular DNA parts that are known to cause ionizing radiation. One of the main advantages of using diode lasers is the ability to interact with diseased tissues selectively and precisely. Lasers enables the clinician to decrease the quantity of bacteria and other pathogens in the surgical area and in the case of soft tissue measures, attain excellent haemostasis with the decreased precondition for sutures.

\section{In contrast to conventional surgery, the claimed benefits of lasers include}

1. Increased coagulation yielding a dry surgical field and improved visualization

2. The ability to negotiate curvatures and folds within tissue contours;

3. Tissue surface disinfection and consequently, decrease in bacteraemia;

4. Reduced distension, edema, and scarring;

5. Decreased pain;

6. Faster healing response; and

7. Increased patient appreciation.

Once laser cutting is in progress, small blood and lymphatic vessels are impassable owing to the heat produced, thus decreasing or eliminating blood flow and edema.The denatured proteins restrained tissue and plasma are the source of "coagulum" layer, that is formed by laser action and protects the wound from bacterial or frictional properties. The diode laser, does not cause any harmful effects on the root surface.Therefore it can be performed safely close to dental hard tissue. ${ }^{12}$

There was definitely no bleeding at all during the procedure. Similarly, the patient experienced no discomfort after surgery and no swelling or other signs of infection were noticed, however other alternative procedures must be accompanied by antibiotic and analgesic administration to minimize postoperative infection and pain.

The superiority of laser over conventional scalpel method has been suggested in many studies, including hemostasis effect, excellent visualization of the operating field, reduce $2 \mathrm{~d}$ operating time, less need for local anesthesia, sterilization of wound site, elimination of suturing, reduction of post-operative edema, pain and minimum scarring. ${ }^{13}$ 
A 2008 article by Kara compared an Nd: YAG laser to conventional scalpel surgery and reported that laser-treated patients reported greater rates of satisfaction, less postoperative pain, and fewer functional problems that impacted chewing or speech ${ }^{14}$ Haytac and Ozcelik compared 20 frenectomy procedures conducted with a $\mathrm{CO} 2$ laser to 20 with standard scalpel surgery and reported less pain after one day and seven days in patients treated with the laser. $^{15}$

Awooda et al. ${ }^{16}$ performed frenectomy in eight patients with diode laser and showed dry and bloodless field during surgery, no post-operative swelling, no pain or discomfort, with normal healing process. Due to its time saving, patient convenience and simple manipulation, the authors recommend the use of laser for soft tissue surgery.

Although emerging research reveals benefits from the use of laser energy, some precautions are required for the laser technique.

1. Practitioners must understand the type of laser being used and the appropriate laser settings and techniques because multiple types of lasers (carbon dioxide, Nd: YAG, diode, etc.) are available.

2. In addition, the clinician and staff should be properly trained for themselves and the patient. in laser safety.

Laser frenectomy procedures are predictably successful, provided the following steps are implemented:

a. Creating a periosteal fenestration at the base of the frenectomy to prevent reattachment of fibers.

b. Removal of all impeding muscle fibers

All laser wavelengths can be used to successfully perform a frenectomy; however, penetration depth for diode and Nd: YAG lasers is much higher $(500 \mu \mathrm{m})$ than for erbium or $\mathrm{CO} 2$ lasers (5 to $40 \mu \mathrm{m}$ ), so the settings need to be closely monitored to prevent thermal damage to the underlying periosteum and bone.

With use of the right parameters (spot size, power, hand speed), one pass of the laser should be sufficient to severe all of the fibers. If multiple passes are needed, care must be taken to prevent excessive lateral thermal necrosis from re-exposure of the already treated tissue. The laser incision is continued to undermine the muscle attachment until the periosteum is reached. All lasers are effective for a frenectomy with settings that the manufacturer suggests. Care should be taken not to char the tissue, with consequent thermal tissue damage. The erbium laser creates a wound that can show some hemorrhage, so it may be necessary to seal the wound with the laser bandage approach.

The other drawbacks of diode lasers are that

1. They are expensive

2. Special equipment required

3. Requires additional skills for operation and limited use.

4. During operation while performing the incision some fumes were released from vaporization of epithelium with a burning smell, which can provoke stress and hurt in the patient, to relieve this complication, it is necessary to operate with a powerful air evacuator.

5. The time required for frenum excision by using diode laser is more than other techniques

6. We can't dissect the entire muscle fibers from the periosteum by using laser so reattachment of frenum may occur. But in conventional scalpel technique we can dissect the entire fibers from the periosteum. So that the chances of reattachment of frenum is very less.

Two months post-operative view (Figure 10) of our case done with laser also shows the reattachment of frenum at the level of mucogingival junction. But not occurred in the case done with conventional technique. Though the laser frenectomy technique has more advantages over the conventional technique, the conventional technique offers the complete dissection of muscle fibers of frenum with less chances of reattachment.

\section{Conclusion}

Frenectomy is a removal of frenum should be considered for potential complications associated with it. Various techniques can be employed for the same but selection of it according to the type of attachment is important for the achievement of proper functional and aesthetic result. Conventional technique and laser have its merits and demerits over other technique but cost-effectiveness also considered before treatment planning.

\section{Source of funding}

None.

\section{Conflict of interest}

None.

\section{References}

1. Mintz SM, Siegel MA, Seider PJ. An overview of oral frena and their association with multiple syndromes and 
nonsyndromic conditions. Oral Surg Oral Med Oral Pathol Oral Radiol Endod 2005;99:321-4.

2. Mirko P, Miroslav S, Lubor M. Significance of the labial frenum attachment in periodontal disease in man. Part I. Classification and epidemiology of the labial frenum attachment. J Periodontol 1974;45:891-4.

3. Olivi G, Chaumanet G, Genovese MD, Beneduce C, Andreana S. Er, Cr: YSGG laser labial frenectomy: A clinical retrospective evaluation of 156 consecutive cases, Laser Therapy. General Dent 2010.

4. Kaur P, Dev YP, Kaushal S, Bhatia A, Vaid R, Sharma R et al. Management Of The Upper Labial Frenum: A Comparison Of Conventional Surgical And Lasers On The Basis Of Visual Analogue Scale On Patients Perception. $J$ Periodontal J Periodontal Med Clin Pract 2014;1(1).

5. Khalid W, Sheeja VS, Sankari M, Jayakumar. The lightsaber is mightier than the sword!-a case of frenectomy using diode laser. Unique J Med Dent Sci 2015;03(01):224.

6. Gargari M, Autili N, Petrone A, Prete V. Using the diode laser in the lower labial frenum removal. Oral Implantol Anno V- N 2-3/2012.

7. Kafas P, Stavrianos C, Jerjes W, Upile T, Vourvachis M, Theodoridis $\mathrm{M}$ et al, Upper-lip laser frenectomy without infiltrated anaesthesia in a paediatric patient: a case report. Cases J 2009;2:7138. 1-3.

8. Tait C. H. Median fraenum of the upper lip and its influence on the spacing of upper central incisor teeth. Dent J 1929; 25: 116.

9. Broadbent, H. Ontogenic development of occlusion. Angle Orthodll 223:1941.
10. Taylor J.E. Clinical observation relating to the normal and abnormal frenum labii superiors. Am J Orthod Oral Surg 1939; $25: 646$.

11. Devishree, Frenectomy: A Review of the Reports of Surgical Techniques. J Clin Diagn Res 2012;6(9):1587-92.

12. Mangalekar SB, Kaushal L, Johnson L, Soni A, Thakur P. Maxillary labial frenectomy using diode laser and classical technique: A case report. Int J Oral Health Med Res 2015;2:48-50.

13. Kotlow L. Diagnosis and treatment of ankyloglossia and tied maxillary frenum in infants using Er.YAG and 1064 diode lasers. Eur Arch Paediatr Dent 2011;12:106-12.

14. Kara C. Evaluation of patient perceptions of frenectomy: A comparison of Nd: YAG laser and conventional techniques. Photomed Laser Surg 2008:26(2):147-52.

15. Haytac MC, Ozcelik O. Evaluation of patient perceptions after frenectomy operations: A comparison of carbon die oxide laser and scalpel techniques. $J$ Periodontol 2006;77(11):1815-19.

16. Awooda EM,Osman B,Nadia A Yahia.Use of Diode Laser (810)nm in frenectomy. Sudan JMS 2007:2(1)

How to cite: Archana N.V*, Paul J, D'Lima JP, parackal ST, Thomas D, Akhil S. Maxillary labial frenectomy by using conventional technique and laser Report of two cases. Int Dent $J$ Student Res 2020;8(1):27-32. 